\title{
Supercompactness and partial level by level equivalence between strong compactness and strongness
}

\author{
by
}

\author{
Arthur W. Apter (New York)
}

\begin{abstract}
We force and construct a model containing supercompact cardinals in which, for any measurable cardinal $\delta$ and any ordinal $\alpha$ below the least beth fixed point above $\delta$, if $\delta^{+\alpha}$ is regular, $\delta$ is $\delta^{+\alpha}$ strongly compact iff $\delta$ is $\delta+\alpha+1$ strong, except possibly if $\delta$ is a limit of cardinals $\gamma$ which are $\delta^{+\alpha}$ strongly compact. The choice of the least beth fixed point above $\delta$ as our bound on $\alpha$ is arbitrary, and other bounds are possible.
\end{abstract}

1. Introduction and preliminaries. In [1], the following theorem was proven.

TheOREM 1. Let $V \vDash " Z F C+\kappa$ is supercompact + There is no pair of cardinals $\delta<\lambda$ such that $\delta$ is $\lambda$ supercompact and $\lambda$ is measurable". There is then a partial ordering $\mathbb{P} \subseteq V$ such that $V^{\mathbb{P}} \vDash " Z F C+G C H+$ There is no pair of cardinals $\delta<\lambda$ such that $\delta$ is $\lambda$ supercompact and $\lambda$ is measurable $+\kappa$ is both the least strongly compact and least strong cardinal (so $\kappa$ is not $2^{\kappa}$ supercompact $)+$ No cardinal $\lambda>\kappa$ is measurable + For $\delta<\kappa$, if $\delta^{+\alpha}$ is regular, then $\delta$ is $\delta^{+\alpha}$ strongly compact iff $\delta$ is $\delta+\alpha+1$ strong".

This theorem provides a counterpoint to the main result of [5], which is as follows.

THEOREM 2. Let $V \vDash " Z F C+\mathfrak{K} \neq \emptyset$ is the class of supercompact cardinals". There is then a partial ordering $\mathbb{P} \subseteq V$ such that $V^{\mathbb{P}} \vDash$ "ZFC+ $G C H+\mathfrak{K}$ is the class of supercompact cardinals + For every pair of regular cardinals $\kappa<\lambda, \kappa$ is $\lambda$ strongly compact iff $\kappa$ is $\lambda$ supercompact, except possibly if $\kappa$ is a limit of cardinals $\delta$ which are $\lambda$ supercompact".

2000 Mathematics Subject Classification: 03E35, 03E55.

Key words and phrases: supercompact cardinal, strongly compact cardinal, strong cardinal, measurable cardinal, non-reflecting stationary set of ordinals, level by level equivalence between strong compactness and supercompactness, level by level equivalence between strong compactness and strongness. 
Whenever the conclusions of Theorem 2 are true, we will say that level by level equivalence between strong compactness and supercompactness holds. Whenever the conclusions of Theorem 1 are true, we will say that level by level equivalence between strong compactness and strongness holds. Observe that in any model witnessing the conclusions of Theorem 2, the KimchiMagidor property [13] holds, i.e., the strongly compact and supercompact cardinals coincide, except possibly at measurable limit points.

Notice that in the model constructed for Theorem 1, there are no supercompact cardinals. In fact, the number of large cardinals in the universe witnessing the conclusions of Theorem 1 is severely restricted. This raises the following questions: Is it possible to get a model in which there is level by level equivalence between strong compactness and strongness and in which there are supercompact cardinals? More generally, is it possible to get a model in which there is level by level equivalence between strong compactness and strongness and in which there is more than one strongly compact cardinal?

The purpose of this paper is to provide a partial affirmative answer to the first of the preceding questions. Specifically, we prove the following theorem.

THEOREM 3. Let $V \vDash " Z F C+\mathfrak{K} \neq \emptyset$ is the class of supercompact cardinals". There is then a partial ordering $\mathbb{P} \subseteq V$ such that $V^{\mathbb{P}} \vDash " Z F C+$ $G C H+\mathfrak{K}$ is the class of supercompact cardinals + The strongly compact and supercompact cardinals coincide, except possibly at measurable limit points + For any measurable cardinal $\delta$ and any $\alpha$ below the least beth fixed point above $\delta$, if $\delta^{+\alpha}$ is regular, $\delta$ is $\delta^{+\alpha}$ strongly compact iff $\delta$ is $\delta+\alpha+1$ strong, except possibly if $\delta$ is a limit of cardinals $\gamma$ which are $\delta^{+\alpha}$ strongly compact".

The choice of the least beth fixed point above $\delta$ as the bound on $\alpha$ in Theorem 3 is done purely as a matter of convenience. Larger bounds on $\alpha$ are also possible. This will be discussed in greater detail at the end of this paper.

We note that by Lemma 1.1 of [3] and the succeeding remark, it is impossible for the least measurable cardinal $\delta$ which is a limit of cardinals $\gamma$ which are either $\delta^{+}$strongly compact or $\delta^{+}$supercompact to be $\delta+2$ strong. This generalizes a result of Menas [14], who also showed that this cardinal $\delta$ must be $\delta^{+}$strongly compact. Thus, when there are large enough cardinals in the universe, it is impossible for there to be a precise level by level equivalence between strong compactness and strongness. (By Lemma 1.2 of [1], a precise level by level equivalence between strong compactness and strongness in the sense that $\delta$ is $\delta^{+\alpha}$ strongly compact iff $\delta$ is $\delta+\alpha+1$ strong for arbitrary $\alpha$ is impossible if there are supercompact cardinals in the universe.)

We observe also that in any model witnessing the conclusions of Theorem 3 , level by level equivalence between strong compactness and super- 
compactness must fail. To see this, note that by either Proposition 26.11 of [12] or Lemma 2.1 of [4], if $\delta$ is $2^{\delta}$ supercompact, $\{\gamma<\delta: \gamma$ is superstrong with target $\delta\}$ is unbounded in $\gamma$. Any such $\gamma$ will of course be $\gamma+\alpha$ strong for every $\alpha$ below the least beth fixed point above $\gamma$. Thus, in a universe in which the conclusions of Theorem 3 hold, below the least cardinal $\kappa$ which is $2^{\kappa}=\kappa^{+}$supercompact, there will be many cardinals $\gamma$ which are $\gamma^{+\alpha}$ strongly compact but not $\gamma^{+\alpha}$ supercompact, where $\alpha$ is any ordinal below the least beth fixed point above $\gamma$.

Before presenting the proof of our theorem, we briefly mention some preliminary information. Essentially, our notation and terminology are standard, and when this is not the case, this will be clearly noted. For $\alpha<\beta$ ordinals, $[\alpha, \beta],[\alpha, \beta),(\alpha, \beta]$, and $(\alpha, \beta)$ are as in standard interval notation.

When forcing, $q \geq p$ will mean that $q$ is stronger than $p$. If $G$ is $V$ generic over $\mathbb{P}$, we will abuse notation somewhat and use both $V[G]$ and $V^{\mathbb{P}}$ to indicate the universe obtained by forcing with $\mathbb{P}$. If $x \in V[G]$, then $\dot{x}$ will be a term in $V$ for $x$. If moreover $\kappa$ is inaccessible and $\mathbb{P}=\left\langle\left\langle\mathbb{P}_{\alpha}, \dot{\mathbb{Q}}_{\alpha}\right\rangle: \alpha<\kappa\right\rangle$ is an Easton support iteration of length $\kappa$ such that at stage $\alpha$, a non-trivial forcing is done adding a subset of $\delta_{\alpha}$, then we will say that $\delta_{\alpha}$ is in the field of $\mathbb{P}$. We may, from time to time, confuse terms with the sets they denote and write $x$ when we actually mean $\dot{x}$ or $\check{x}$, especially when $x$ is some variant of the generic set $G$, or $x$ is in the ground model $V$.

Let $\kappa$ be a regular cardinal. The partial ordering $\mathbb{P}$ is $\kappa$-directed closed if for every cardinal $\delta<\kappa$ and every directed set $\left\langle p_{\alpha}: \alpha<\delta\right\rangle$ of elements of $\mathbb{P}$ (where $\left\langle p_{\alpha}: \alpha<\delta\right\rangle$ is directed if for any two distinct elements $p_{\varrho}, p_{\nu} \in$ $\left\langle p_{\alpha}: \alpha<\delta\right\rangle, p_{\varrho}$ and $p_{\nu}$ have a common upper bound of the form $p_{\sigma}$ ) there is an upper bound $p \in \mathbb{P}$. $\mathbb{P}$ is $\kappa$-strategically closed if in the two-person game in which the players construct an increasing sequence $\left\langle p_{\alpha}: \alpha \leq \kappa\right\rangle$, where player I plays odd stages and player II plays even and limit stages (choosing the trivial condition at stage 0), player II has a strategy which ensures the game can always be continued. Note that if $\mathbb{P}$ is $\kappa$-strategically closed and $f: \kappa \rightarrow V$ is a function in $V^{\mathbb{P}}$, then $f \in V$. $\mathbb{P}$ is $<\kappa$-strategically closed if $\mathbb{P}$ is $\delta$-strategically closed for all cardinals $\delta<\kappa$. $\mathbb{P}$ is $\prec \kappa$-strategically closed if in the two-person game in which the players construct an increasing sequence $\left\langle p_{\alpha}: \alpha<\kappa\right\rangle$, where player I plays odd stages and player II plays even and limit stages, player II has a strategy which ensures the game can always be continued.

Suppose now that $\kappa$ is a Mahlo cardinal. A partial ordering $\mathbb{P}(\kappa)$ to be used in the proof of Theorem 3 is the partial ordering for adding a non-reflecting stationary set of ordinals of a certain type to $\kappa$. Specifically, $\mathbb{P}(\kappa)=\{p:$ For some $\alpha<\kappa, p: \alpha \rightarrow\{0,1\}$ is a characteristic function of $S_{p}$, a subset of $\alpha$ not stationary at its supremum nor having any initial segment which is stationary at its supremum, such that if $\beta<\sup \left(S_{p}\right)$ is 
inaccessible, then $S_{p}-S_{p} \cap \beta$ is composed of ordinals of cofinality at least $\beta$, ordered by $q \geq p$ iff $q \supseteq p$ and $S_{p}=S_{q} \cap \sup \left(S_{p}\right)$, i.e., $S_{q}$ is an end extension of $S_{p}$. By Lemmas 1.1-1.3 of [2] and the succeeding remarks, we have the following facts:

1. $\mathbb{P}(\kappa)$ is $\prec \kappa$-strategically closed.

2. Forcing with $\mathbb{P}(\kappa)$ adds a non-reflecting stationary set of ordinals to $\kappa$.

3. For any inaccessible cardinal $\delta<\kappa$, the partial ordering $\mathbb{P}(\kappa / \delta)=$ $\{p \in \mathbb{P}(\kappa): p$ is either the characteristic function of the empty set, or is such that $S_{p}$ contains an ordinal above $\left.\delta\right\}$ with the inherited partial ordering is dense in $\mathbb{P}(\kappa)$ and is $\delta$-directed closed.

In addition, since $\kappa$ is Mahlo, it easily follows that $|\mathbb{P}(\kappa)|=\kappa$. By (1) above therefore, if GCH holds in our ground model, it then easily follows that forcing with $\mathbb{P}(\kappa)$ preserves GCH.

We mention that we are assuming familiarity with the large cardinal notions of measurability, strongness, superstrongness, strong compactness, and supercompactness. Interested readers may consult [12] for further details. We note explicitly that the cardinal $\kappa$ is $<\lambda$ supercompact if $\kappa$ is $\delta$ supercompact for every cardinal $\delta<\lambda$. Also, unlike [12], we will say that the cardinal $\kappa$ is $\lambda$ strong for $\lambda>\kappa$ if there is $j: V \rightarrow M$ an elementary embedding having critical point $\kappa$ such that $j(\kappa)>\left|V_{\lambda}\right|$ and $V_{\lambda} \subseteq M$. If $\left|V_{\lambda}\right|$ is regular, then we may assume that $M^{\kappa} \subseteq M$ as well. In addition, it is easily seen that any cardinal $\kappa$ which is $\kappa^{+\alpha}$ supercompact is $\kappa+\alpha+1$ strong.

2. The proof of Theorem 3. Let $V \vDash " \mathrm{ZFC}+\mathfrak{K}$ is the class of supercompact cardinals". Without loss of generality, by first forcing GCH and then forcing with the partial ordering of [5], we may also assume that GCH and level by level equivalence between strong compactness and supercompactness hold in $V$.

We are now in a position to define the partial ordering $\mathbb{P}$ that will be used in the proof of Theorem 3. Let $\mathfrak{D}$ be the collection of $V$-measurable cardinals $\delta$ such that for some $\alpha$ below the least beth fixed point above $\delta$, in $V, \delta^{+\alpha}$ is regular, $\delta$ is $\delta+\alpha+1$ strong, yet $\delta$ is not $\delta^{+\alpha}$ supercompact. $\mathbb{P}$ is then taken as the Easton support iteration which begins by adding a Cohen subset of $\omega$ and then adds, to every $\delta \in \mathfrak{D}$, a non-reflecting stationary set of ordinals using the partial ordering $\mathbb{P}(\delta)$ defined in Section 1. By the usual Easton arguments, regardless of whether $\mathbb{P}$ is a set or a proper class, $V^{\mathbb{P}} \vDash$ ZFC. Further, it is easily shown by an induction similar to the one given in the proof of Lemma 8 of [5] (see also [1]) that $V^{\mathbb{P}} \vDash$ GCH and forcing with $\mathbb{P}$ preserves cardinals and cofinalities. This means that henceforth, without fear of ambiguity, we will write $\delta^{+\alpha}$ without specifying explicitly whether we are working in $V, V^{\mathbb{P}}$, or some $\bar{V}$ with $V \subseteq \bar{V} \subseteq V^{\mathbb{P}}$. 


\section{LEMMA 2.1. $V^{\mathbb{P}} \vDash$ "K is the class of supercompact cardinals".}

Proof. The argument we give is very similar to the one presented in the proof of Lemma 3.1 of $[2]$. Write $\mathbb{P}=\mathbb{P}^{\prime} * \dot{\mathbb{P}}^{\prime \prime}$, where $\left|\mathbb{P}^{\prime}\right|=\omega, \mathbb{P}^{\prime}$ is nontrivial, and $\vdash_{\mathbb{P}^{\prime}}$ " $\dot{\mathbb{P}} "$ is $\aleph_{1}$-strategically closed". In the terminology of $[9]-[11]$, $\mathbb{P}$ "admits a gap at $\aleph_{1}$ ", so by the results of [9]-[11], any supercompact cardinal in $V^{\mathbb{P}}$ had to have been supercompact in $V$. This means the proof of Lemma 2.1 will be complete once we have shown that $V^{\mathbb{P}} \vDash$ "If $\kappa \in \mathfrak{K}$, then $\kappa$ is supercompact".

To do this, fix $\kappa \in \mathfrak{K}$. Let $A$ be an arbitrary (possibly empty) set of $V$-measurable cardinals above $\kappa$. Take $\eta=\max (\sup (A), \kappa)$, and define $\eta^{*}$ as the least $V$-measurable cardinal above $\eta$ if this cardinal exists, or the class of all ordinals if no cardinal above $\eta$ is measurable in $V$. Let $\lambda \in\left(\eta, \eta^{*}\right)$ be any successor cardinal above the least beth fixed point above $\eta$. Let $\gamma=\left|2^{[\lambda]^{<\kappa}}\right|$, and fix $j: V \rightarrow M$ an elementary embedding witnessing the $\gamma$ supercompactness of $\kappa$.

Write $\mathbb{P}=\mathbb{P}^{0} * \dot{\mathbb{P}}^{1} * \dot{\mathbb{P}}^{2}$, where $\mathbb{P}^{0}$ is the portion of $\mathbb{P}$ defined through stage $\kappa, \dot{\mathbb{P}}^{1}$ is a term for the portion of $\mathbb{P}$ defined between stages $\kappa$ and $\lambda$, and $\dot{\mathbb{P}}^{2}$ is a term for the rest of $\mathbb{P}$. By the definition of $\mathbb{P}$, it will be the case that $\vdash_{\mathbb{P}^{0} * \mathbb{P}^{1}}$ " $\dot{\mathbb{P}}^{2}$ is $\gamma$-strategically closed". Thus, since $\lambda$ may be chosen arbitrarily large, to prove Lemma 2.1, it will suffice to show that $V^{\mathbb{P}^{0} * \dot{\mathbb{P}}^{1}} \vDash$ " $\kappa$ is $\lambda$ supercompact".

If this is not the case, then let $p=\left\langle p_{0}, \dot{p}_{1}\right\rangle \in \mathbb{P}^{0} * \dot{\mathbb{P}}^{1}$ be such that $p \Vdash$ " $\kappa$ is not $\lambda$ supercompact". By using Lemma 1.2 of [2] if necessary to find the necessary terms to extend coordinatewise, we assume without loss of generality that each non-trivial coordinate of $p_{1}$ is a term for a condition in the appropriate $\mathbb{P}(\delta / \kappa)$.

Let $G_{0}$ be $V$-generic over $\mathbb{P}^{0}$ such that $p_{0} \in G_{0}$. Working in $V\left[G_{0}\right]$ and once again using Lemma 1.2 of [2], let $\mathbb{P}^{3}$ be the Easton support iteration of partial orderings which, for every $V$-measurable cardinal $\delta \in(\kappa, \lambda)$ which is an element of $\mathfrak{D}$, add non-reflecting stationary sets of ordinals using $\mathbb{P}(\delta / \kappa)$.

Note now that if $G_{1}$ is $V\left[G_{0}\right]$-generic over $\mathbb{P}^{3}$ and $p_{1} \in G_{1}$, then $G_{1}$ must also generate a $V\left[G_{0}\right]$-generic filter $G_{1}^{*}$ over $\mathbb{P}^{1}$. To see this, it clearly suffices to show that $G_{1}$ meets all dense open subsets of $\mathbb{P}^{1}$ above $p_{1}$. If $D$ is such a set, then let $D_{1}=\left\{q \in \mathbb{P}^{3}: q\right.$ extends some element of $\left.D\right\}$. $D_{1}$ is clearly open. If $q \in \mathbb{P}^{3}$, then $q \in \mathbb{P}^{1}$, so by density, there is $q^{\prime} \geq q, q^{\prime} \in D$. By using Lemma 1.2 of [2] if necessary to find a term which is forced to extend each term denoting a non-trivial coordinate of $q^{\prime}$ to a term for an element of the appropriate $\mathbb{P}(\delta / \kappa)$, we obtain $q^{\prime \prime} \geq q^{\prime} \geq q, q^{\prime \prime} \in D_{1}$. Thus, $G_{1}$ meets $D_{1}$ and hence meets $D$, so $G_{1}$ generates a $V\left[G_{0}\right]$-generic filter $G_{1}^{*}$ over $\mathbb{P}^{1}$.

By the definition of $\mathbb{P}$ and the closure properties of $M, j\left(\mathbb{P}^{0} * \dot{\mathbb{P}}^{1}\right)=\mathbb{P}^{0} *$ $\dot{\mathbb{P}}^{1} * \dot{\mathbb{Q}} * \dot{\mathbb{R}}$, where $\dot{\mathbb{Q}}$ is a term for the portion of $j\left(\mathbb{P}^{0} * \dot{\mathbb{P}}^{1}\right)$ defined in $M$ between 
stages $\lambda$ and $j(\kappa)$, and $\dot{\mathbb{R}}$ is a term for $j\left(\dot{\mathbb{P}}^{1}\right)$, i.e., the portion of $j\left(\mathbb{P}^{0} * \dot{\mathbb{P}}^{1}\right)$ defined in $M$ between stages $j(\kappa)$ and $j(\lambda)$. If $G_{1}$ is $V\left[G_{0}\right]$-generic over $\mathbb{P}^{3}$ and $p_{1} \in G_{1}$, then by the preceding paragraph, $G_{1}$ generates a $V\left[G_{0}\right]$-generic filter $G_{1}^{*}$ over $\mathbb{P}^{1}$. We can therefore take $G_{2}$ as a $V\left[G_{0}\right]\left[G_{1}^{*}\right]$-generic object over $\mathbb{Q}$ and use the usual Easton arguments to infer that $M\left[G_{0}\right]\left[G_{1}^{*}\right]\left[G_{2}\right]$ remains $\gamma$ closed with respect to $V\left[G_{0}\right]\left[G_{1}^{*}\right]\left[G_{2}\right]$ and that $j$ lifts in $V\left[G_{0}\right]\left[G_{1}^{*}\right]\left[G_{2}\right]$ to $j: V\left[G_{0}\right] \rightarrow M\left[G_{0}\right]\left[G_{1}^{*}\right]\left[G_{2}\right]$. Further, since $G_{1} \subseteq G_{1}^{*}$ and $G_{1}$ is $V\left[G_{0}\right]$-generic over a partial ordering (namely $\mathbb{P}^{3}$ ) that is $\kappa$-directed closed in $V\left[G_{0}\right], j^{\prime \prime} G_{1}$ generates in $V\left[G_{0}\right]\left[G_{1}^{*}\right]\left[G_{2}\right]$ a compatible set of conditions of cardinality smaller than $\gamma<j(\kappa)$ in a partial ordering (namely $j\left(\mathbb{P}^{3}\right)$ ) that is $j(\kappa)$ directed closed in $M\left[G_{0}\right]\left[G_{1}^{*}\right]\left[G_{2}\right]$. Therefore, by the fact $M\left[G_{0}\right]\left[G_{1}^{*}\right]\left[G_{2}\right]$ is $\gamma$ closed with respect to $V\left[G_{0}\right]\left[G_{1}^{*}\right]\left[G_{2}\right]$, we can let $r$ be a master condition for $j^{\prime \prime} G_{1}$ and take $G_{3}$ to be a $V\left[G_{0}\right]\left[G_{1}^{*}\right]\left[G_{2}\right]$-generic object over $j\left(\mathbb{P}^{3}\right)$ containing $r$. By elementarity, it will be the case that $G_{3}$ generates a $V\left[G_{0}\right]\left[G_{1}^{*}\right]\left[G_{2}\right]$ generic object $G_{3}^{*}$ over $\mathbb{R}=j\left(\mathbb{P}^{1}\right)$. As usual, in $V\left[G_{0}\right]\left[G_{1}^{*}\right]\left[G_{2}\right]\left[G_{3}^{*}\right], j$ will then lift to $j: V\left[G_{0}\right]\left[G_{1}^{*}\right] \rightarrow M\left[G_{0}\right]\left[G_{1}^{*}\right]\left[G_{2}\right]\left[G_{3}^{*}\right]$, so $\kappa$ is $\lambda$ supercompact in $V\left[G_{0}\right]\left[G_{1}^{*}\right]\left[G_{2}\right]\left[G_{3}^{*}\right]$. Since $\mathbb{Q} * \dot{\mathbb{R}}$ is $\gamma$-strategically closed in $V\left[G_{0}\right]\left[G_{1}^{*}\right]$, it will be the case that $\kappa$ is $\lambda$ supercompact in $V\left[G_{0}\right]\left[G_{1}^{*}\right]$. This, however, contradicts the fact that $p=\left\langle p_{0}, p_{1}\right\rangle \in G_{0} * G_{1}^{*}$ and $p \Vdash$ " $\kappa$ is not $\lambda$ supercompact". This contradiction completes the proof of Lemma 2.1.

We remark that the proof of Lemma 2.1 actually shows that any cardinal $\delta$ which is in $V$ both regular and a limit of cardinals $\kappa$ which are $<\delta$ supercompact remains in $V^{\mathbb{P}}$ regular and a limit of cardinals $\kappa$ which are $<\delta$ supercompact. To see this, let $\delta>\lambda>\kappa$ be such that $V \vDash " \kappa$ is $<\delta$ supercompact and $\delta$ is regular". Work in $\bar{V}=V_{\delta}$, and take $\mathbb{Q}=\mathbb{P}_{\delta}$. Fix $j: \bar{V} \rightarrow M$ an elementary embedding witnessing the $\lambda$ supercompactness of $\kappa$. The same proof as just given shows that $\bar{V}^{\mathbb{Q}} \vDash$ " $\kappa$ is $\lambda$ supercompact". Since writing $\mathbb{P}=\mathbb{P}_{\delta} * \dot{\mathbb{P}}^{\delta}$ tells us $\Vdash_{\mathbb{P}_{\delta}}$ " $\dot{\mathbb{P}}^{\delta}$ is $<\delta$-strategically closed", we can now immediately infer that $V^{\mathbb{P}_{\delta} * \dot{\mathbb{P}}^{\delta}}=V^{\mathbb{P}} \vDash " \delta$ is regular and $\kappa$ is $<\delta$ supercompact". As $\kappa$ and $\lambda$ were arbitrary, in $V^{\mathbb{P}}, \delta$ is regular and is a limit of $<\delta$ supercompact cardinals.

We note also that the proof of Lemma 2.1 does not necessarily show, however, that if $\lambda>\delta$ and $\delta$ is in $V$ both regular and a limit of cardinals $\kappa$ which are $\lambda$ supercompact, then $\delta$ remains in $V^{\mathbb{P}}$ a limit of cardinals $\kappa$ which are $\lambda$ supercompact. To see this, let $\kappa<\delta$ be $\lambda$ supercompact in $V$, and fix $j: V \rightarrow M$ an elementary embedding witnessing the $\lambda$ supercompactness of $\kappa$. It is possible that the closure properties of $M$ with respect to $V$ are not enough to guarantee that $V$ and $M$ make the same decision as to whether a non-reflecting stationary set of ordinals must be added to $\delta$. If the decisions differ, then the proof of Lemma 2.1 suitably modified will not remain valid. 
LEMMA 2.2. $V^{\mathbb{P}} \vDash$ "The strongly compact and supercompact cardinals coincide, except possibly at measurable limit points".

Proof. Suppose $V^{\mathbb{P}} \vDash$ " $\delta$ is strongly compact". Write $\mathbb{P}=\mathbb{P}_{\delta} * \dot{\mathbb{P}}^{\delta}$. Using this factorization, it is easy to see by $\mathbb{P}$ 's definition that $\mathbb{P}$ is, in the terminology of [10] and [11], "mild with respect to $\delta$ ". This means that any set of ordinals $x$ in $V^{\mathbb{P}}$ of size below $\delta$ has a "nice" name $\tau$ in $V$ of size below $\delta$, i.e., there is a set $y$ in $V,|y|<\delta$, such that any ordinal forced by a condition in $\mathbb{P}$ to be in $\tau$ is an element of $y$. Since we have already seen that $\mathbb{P}$ "admits a gap at $\aleph_{1}$ ", by the results of [10] and [11], $\delta$ had to have been strongly compact in $V$. Thus, in $V, \delta$ is either an element of $\mathfrak{K}$ or a measurable limit of elements of $\mathfrak{K}$, so by Lemma 2.1 , in $V^{\mathbb{P}}, \delta$ is either supercompact or a measurable limit of supercompact cardinals. This completes the proof of Lemma 2.2.

LEMMA 2.3. $V^{\mathbb{P}} \vDash$ "For any measurable cardinal $\delta$ and any $\alpha$ below the least beth fixed point above $\delta$, if $\delta^{+\alpha}$ is regular and $\delta$ is $\delta+\alpha+1$ strong, then $\delta$ is $\delta^{+\alpha}$ strongly compact".

Proof. We begin by observing we may assume without loss of generality that $\alpha>0$. This is since if $\delta$ is $\delta+0+1=\delta+1$ strong, $\delta$ is measurable, so by definition, $\delta$ is both $\delta^{+0}=\delta$ strongly compact and $\delta$ supercompact.

Suppose now that $\alpha>0, \alpha$ is below the least beth fixed point above $\delta$, and $V^{\mathbb{P}} \vDash " \delta$ is $\delta+\alpha+1$ strong and $\delta^{+\alpha}$ is regular". Since we have already observed that $\mathbb{P}$ "admits a gap at $\aleph_{1}$ ", by the results of [9]-[11], $\delta$ had to have been $\delta+\alpha+1$ strong in $V$ as well. Therefore, $\delta$ was $\delta^{+\alpha}$ supercompact in $V$ also, since otherwise, the definition of $\mathbb{P}$ tells us we can write $\mathbb{P}=$ $\mathbb{P}_{\delta} * \dot{\mathbb{P}}(\delta) * \dot{\mathbb{P}} \delta$. We may then infer that $\vdash_{\mathbb{P}_{\delta}} " \dot{\mathbb{P}}(\delta)$ adds a non-reflecting stationary set of ordinals to $\delta$ " and $\Vdash_{\mathbb{P}_{\delta} * \dot{\mathbb{P}}(\delta)}$ " $\dot{\mathbb{P}} \delta$ is $\eta$-strategically closed for $\eta$ the least inaccessible above $\delta "$. This means that $V^{\mathbb{P}_{\delta} * \dot{\mathbb{P}}(\delta) * \dot{\mathbb{P}}^{\delta}}=V^{\mathbb{P}} \vDash " \delta$ contains a non-reflecting stationary set of ordinals and hence is not weakly compact", a contradiction to the fact that $V^{\mathbb{P}} \vDash$ " $\delta$ is $\delta+\alpha+1$ strong". Thus, we can actually write $\mathbb{P}=\mathbb{P}_{\delta} * \dot{\mathbb{P}}^{\delta}$, where $\Vdash_{\mathbb{P}_{\delta}}$ " $\dot{\mathbb{P}}^{\delta}$ is $\eta$-strategically closed for $\eta$ the least inaccessible above $\delta$ ". Hence, since $\alpha$ is below the least beth fixed point above $\delta$, to show that $V^{\mathbb{P}} \vDash$ " $\delta$ is $\delta^{+\alpha}$ strongly compact", it suffices to show that $V^{\mathbb{P}_{\delta}} \vDash$ " $\delta$ is $\delta^{+\alpha}$ strongly compact".

To show $V^{\mathbb{P}_{\delta}} \vDash " \delta$ is $\delta^{+\alpha}$ strongly compact", we use an argument of Magidor for the preservation of strong compactness. Although the essentials of this argument can be found in [2] and [4] (as well as elsewhere), for completeness and for the benefit of readers, we give the argument here as well. Let $\lambda=\delta^{+\alpha}$, and let $k_{1}: V \rightarrow M$ be an elementary embedding witnessing the $\lambda$ supercompactness of $\delta$ such that $M \vDash$ " $\delta$ is not $\lambda$ supercompact". Since $M \vDash$ " $\delta$ is measurable", we may choose a normal ultrafilter of Mitchell order 0 over $\delta$ such that $k_{2}: M \rightarrow N$ is an elementary embedding witness- 
ing the measurability of $\delta$ definable in $M$ with $N \vDash$ " $\delta$ is not measurable". It is the case that if $k: V \rightarrow N$ is an elementary embedding with critical point $\delta$ and for any $x \subseteq N$ with $|x| \leq \lambda$, there is some $y \in N$ such that $x \subseteq y$ and $N \vDash "|y|<k(\delta)$ ", then $k$ witnesses the $\lambda$ strong compactness of $\delta$. Using this fact, it is easily verifiable that $j=k_{2} \circ k_{1}$ is an elementary embedding witnessing the $\lambda$ strong compactness of $\delta$. We show that $j$ lifts to $j: V^{\mathbb{P}_{\delta}} \rightarrow N^{j\left(\mathbb{P}_{\delta}\right)}$. Since this lifted embedding witnesses the $\lambda$ strong compactness of $\delta$ in $V^{\mathbb{P}_{\delta}}$, this proves Lemma 2.3.

To do this, write $j\left(\mathbb{P}_{\delta}\right)$ as $\mathbb{P}_{\delta} * \dot{\mathbb{Q}}^{0} * \dot{\mathbb{R}}^{0}$, where $\dot{\mathbb{Q}}^{0}$ is a term for the portion of $j\left(\mathbb{P}_{\delta}\right)$ between $\delta$ and $k_{2}(\delta)$ and $\dot{\mathbb{R}}^{0}$ is a term for the rest of $j\left(\mathbb{P}_{\delta}\right)$, i.e., the part above $k_{2}(\delta)$. Note that since $N \vDash$ " $\delta$ is not measurable", $\delta \notin$ field $\left(\dot{\mathbb{Q}}^{0}\right)$. Thus, the field of $\dot{\mathbb{Q}}^{0}$ is composed of all $N$-measurable cardinals $\gamma \in\left(\delta, k_{2}(\delta)\right.$ ] for which in $N$, for some $\beta$ below the least beth fixed point above $\gamma, \gamma^{+\beta}$ is regular, $\gamma$ is $\gamma+\beta+1$ strong, yet $\gamma$ is not $\gamma^{+\beta}$ supercompact. This means $k_{2}(\delta) \in \operatorname{field}\left(\dot{\mathbb{Q}}^{0}\right)$, since by either Proposition 26.11 of [12] or Lemma 2.1 of [4], $M \vDash$ " $\delta$ is superstrong" yet $M \vDash$ " $\delta$ is not $\delta^{+\alpha}$ supercompact and $\alpha$ is below the least beth fixed point above $\delta$ ", so by elementarity, $N \vDash$ "There is some $\beta$ below the least beth fixed point above $j(\delta)$ for which $j(\delta)$ is $j(\delta)+\beta+1$ strong yet $j(\delta)$ is not $(j(\delta))^{+\beta}$ supercompact". Also, the field of $\dot{\mathbb{R}}^{0}$ is composed of all $N$-measurable cardinals $\gamma \in\left(k_{2}(\delta), k_{2}\left(k_{1}(\delta)\right)\right)$ for which in $N$, for some $\beta$ below the least beth fixed point above $\gamma, \gamma^{+\beta}$ is regular, $\gamma$ is $\gamma+\beta+1$ strong, yet $\gamma$ is not $\gamma^{+\beta}$ supercompact.

Let $G_{0}$ be $V$-generic over $\mathbb{P}_{\delta}$. We construct in $V\left[G_{0}\right]$ an $N\left[G_{0}\right]$-generic object $G_{1}$ over $\mathbb{Q}^{0}$ and an $N\left[G_{0}\right]\left[G_{1}\right]$-generic object $G_{2}$ over $\mathbb{R}^{0}$. Since $\mathbb{P}_{\delta}$ is an Easton support iteration of length $\delta$, a direct limit is taken at stage $\delta$, and no forcing is done at stage $\delta$, the construction of $G_{1}$ and $G_{2}$ automatically guarantees that $j^{\prime \prime} G_{0} \subseteq G_{0} * G_{1} * G_{2}$. This means that $j: V \rightarrow N$ lifts to $j: V\left[G_{0}\right] \rightarrow N\left[G_{0}\right]\left[G_{1}\right]\left[G_{2}\right]$ in $V\left[G_{0}\right]$.

To build $G_{1}$, note that since $k_{2}$ is generated by an ultrafilter $\mathcal{U}$ over $\delta$ and since in both $V$ and $M, 2^{\delta}=\delta^{+},\left|k_{2}\left(\delta^{+}\right)\right|=\left|k_{2}\left(2^{\delta}\right)\right|=\mid\left\{f: f: \delta \rightarrow \delta^{+}\right.$ is a function $\}|=|\left[\delta^{+}\right]^{\delta} \mid=\delta^{+}$. Thus, as $N\left[G_{0}\right] \vDash "\left|\wp\left(\mathbb{Q}^{0}\right)\right|=k_{2}\left(2^{\delta}\right)$ ", we can let $\left\langle D_{\beta}: \beta<\delta^{+}\right\rangle$enumerate in $V\left[G_{0}\right]$ the dense open subsets of $\mathbb{Q}^{0}$ found in $N\left[G_{0}\right]$. For the purpose of the construction of $G_{1}$ to be given below, we further assume that for every dense open subset $D \subseteq \mathbb{Q}^{0}$ present in $N\left[G_{0}\right]$, for some odd ordinal $\gamma+1, D=D_{\gamma+1}$. Since the $\delta$ closure of $N$ with respect to either $M$ or $V$ implies the least element of the field of $\mathbb{Q}^{0}$ is above $\delta^{+}$, the definition of $\mathbb{Q}^{0}$ as given above implies that $N\left[G_{0}\right] \vDash " \mathbb{Q}^{0}$ is $\prec \delta^{+}$-strategically closed". By the fact the standard arguments show that forcing with the $\delta$ c.c. partial ordering $\mathbb{P}_{\delta}$ preserves that $N\left[G_{0}\right]$ remains $\delta$-closed with respect to either $M\left[G_{0}\right]$ or $V\left[G_{0}\right], \mathbb{Q}^{0}$ is $\prec \delta^{+}$-strategically closed in both $M\left[G_{0}\right]$ and $V\left[G_{0}\right]$. 
We can now construct $G_{1}$ in either $M\left[G_{0}\right]$ or $V\left[G_{0}\right]$ as follows. Players I and II play a game of length $\delta^{+}$. The initial pair of moves is generated by player II choosing the trivial condition $q_{0}$ and player I responding by choosing $q_{1} \in D_{1}$. Then, at an even stage $\beta+2$, player II picks $q_{\beta+2} \geq q_{\beta+1}$ by using some fixed strategy $\mathcal{S}$, where $q_{\beta+1}$ was chosen by player I to be such that $q_{\beta+1} \in D_{\beta+1}$ and $q_{\beta+1} \geq q_{\beta}$. If $\beta$ is a limit ordinal, player II uses $\mathcal{S}$ to pick $q_{\beta}$ extending each $q_{\gamma}$ for $\gamma<\beta$. By the $\prec \delta^{+}$-strategic closure of $\mathbb{Q}^{0}$ in both $M\left[G_{0}\right]$ and $V\left[G_{0}\right]$, the sequence $\left\langle q_{\beta}: \beta<\delta^{+}\right\rangle$as just described exists. By construction, $G_{1}=\left\{p \in \mathbb{Q}^{0}: \exists \beta<\delta^{+}\left[q_{\beta} \geq p\right]\right\}$ is our $N\left[G_{0}\right]$-generic object over $\mathbb{Q}^{0}$.

It remains to construct in $V\left[G_{0}\right]$ the desired $N\left[G_{0}\right]\left[G_{1}\right]$-generic object $G_{2}$ over $\mathbb{R}^{0}$. To do this, we first note that as $M \vDash$ " $\delta$ is superstrong but $\delta$ is not $\delta^{+\alpha}$ supercompact and $\alpha$ is below the least beth fixed point above $\delta$ ", we can write $k_{1}\left(\mathbb{P}_{\delta}\right)$ as $\mathbb{P}_{\delta} * \dot{\mathbb{S}}^{0} * \dot{\mathbb{T}}^{0}$, where $\vdash_{\mathbb{P}_{\delta}}$ " $\dot{\mathbb{S}}^{0}=\dot{\mathbb{P}}(\delta)$ ", and $\dot{\mathbb{T}}^{0}$ is a term for the rest of $k_{1}\left(\mathbb{P}_{\delta}\right)$.

Note now that $M \vDash$ "No cardinal $\gamma \in(\delta, \lambda]$ is measurable". Thus, the field of $\dot{\mathbb{T}}^{0}$ is composed only of $M$-measurable cardinals in the interval $\left(\lambda, k_{1}(\delta)\right)$, which implies that in $M, \Vdash_{\mathbb{P}_{\delta} * \dot{\mathbb{S}}^{0}}$ " $\dot{\mathbb{T}}^{0}$ is $\prec \lambda^{+}$-strategically closed". Further, since $V \vDash \mathrm{GCH}$ and $\lambda$ is regular, $\left|[\lambda]^{<\delta}\right|=\lambda$ and $2^{\lambda}=\lambda^{+}$. Therefore, as $k_{1}$ can be assumed to be generated by an ultrafilter $\mathcal{U}$ over $P_{\delta}(\lambda),\left|k_{1}\left(\delta^{+}\right)\right|=\left|2^{k_{1}(\delta)}\right|=\mid\left\{f: f: P_{\delta}(\lambda) \rightarrow \delta^{+}\right.$is a function $\}|=|\left[\delta^{+}\right]^{\lambda} \mid=$ $\left|[\lambda]^{\lambda}\right|=\lambda^{+}$.

Work until otherwise specified in $M$. Consider the "term forcing" partial ordering $\mathbb{T}^{*}$ (see $[8]$ for the first published account of term forcing or $[6$, Section 1.2.5, p. 8]; the notion is originally due to Laver) associated with $\stackrel{\mathbb{T}}{ }^{0}$, i.e., $\tau \in \mathbb{T}^{*}$ iff $\tau$ is a term in the forcing language with respect to $\mathbb{P}_{\delta} * \dot{\mathbb{S}}^{0}$ and $\Vdash_{\mathbb{P}_{\delta} * \dot{\mathbb{S}}^{0}}$ " $\tau \in \dot{\mathbb{T}}^{0}$ ", ordered by $\tau \geq \sigma$ iff $\vdash_{\mathbb{P}_{\delta} * \dot{\mathbb{S}}^{0}}$ " $\tau \geq \sigma$ ". Although $\mathbb{T}^{*}$ as defined is technically a proper class, it is possible to restrict the terms appearing in it to a sufficiently large set-sized collection, with the additional crucial property that any term $\tau$ forced to be in $\dot{\mathbb{T}}^{0}$ is also forced to be equal to an element of $\mathbb{T}^{*}$. As we will show below, this can be done in such a way that $M \vDash "\left|\mathbb{T}^{*}\right|=k_{1}(\delta)$ ".

Clearly, $\mathbb{T}^{*} \in M$. Also, since $\Vdash_{\mathbb{P}_{\delta} * \dot{\mathbb{S}}^{0}}$ " $\dot{\mathbb{T}}^{0}$ is $\prec \lambda^{+}$-strategically closed", it can easily be verified that $\mathbb{T}^{*}$ itself is $\prec \lambda^{+}$-strategically closed in $M$ and, since $M^{\lambda} \subseteq M$, in $V$ as well.

To show that we may restrict the number of terms so that $M \vDash "\left|\mathbb{T}^{*}\right|=$ $k_{1}(\delta)$ ", we observe that since $\Vdash_{\mathbb{P}_{\delta} * \dot{\mathbb{S}}^{0}}$ " $\left|\dot{\mathbb{T}}^{0}\right|=k_{1}(\delta)$ ", there is a set $\left\{\tau_{\beta}\right.$ : $\left.\beta<k_{1}(\delta)\right\}$ of terms such that for any other term $\tau$, if $\vdash_{\mathbb{P}_{\delta} * \dot{S}^{0}}$ " $\tau \in \dot{\mathbb{T}}$ ", then there is a dense set of conditions in $\mathbb{P}_{\delta} * \dot{\mathbb{S}}^{0}$ forcing " $\tau=\tau_{\beta}$ " for various $\beta$. While $\left\{\tau_{\beta}: \beta<k_{1}(\delta)\right\}$ may not itself be adequate, we enlarge it by choosing, for each maximal antichain $A \subseteq \mathbb{P}_{\delta} * \dot{\mathbb{S}}^{0}$ and each function 
$s: A \rightarrow\left\{\tau_{\beta}: \beta<k_{1}(\delta)\right\}$, a term $\tau_{s}$ such that $p \Vdash$ " $\tau_{\beta}=\tau_{s(p)}$ " for each $p \in A$. $\tau_{s}$ exists using arguments from elementary forcing. Let $\mathbb{T}^{*}$ be the collection of all such terms $\tau_{s}$, ranging over all maximal antichains of $\mathbb{P}_{\delta} * \dot{\mathbb{S}}^{0}$. Since $M \vDash "\left|\mathbb{P}_{\delta} * \dot{\mathbb{S}}^{0}\right|<k_{1}(\delta)$ and $k_{1}(\delta)$ is measurable", the number of such terms in $M$ is $k_{1}(\delta)$. Finally, if $\vdash_{\mathbb{P}_{\delta} * \dot{\mathbb{S}}^{0}}$ " $\tau \in \dot{\mathbb{T}}$ ", then once again, elementary forcing arguments establish that for some $s, \Vdash_{\mathbb{P}_{\delta} * \dot{\mathbb{S}}^{0}}$ " $\tau=\tau_{s}$ ". Therefore, as we now know that $M \vDash "\left|\mathbb{T}^{*}\right|=k_{1}(\delta)$ ", and as $M \vDash " 2^{k_{1}(\delta)}=\left(k_{1}(\delta)\right)^{+}=k_{1}\left(\delta^{+}\right)$", this means we can let $\left\langle D_{\beta}: \beta<\lambda^{+}\right\rangle$enumerate in $V$ the dense open subsets of $\mathbb{T}^{*}$ found in $M$, such that as before, for every dense open subset $D \subseteq \mathbb{T}^{*}$ present in $M$, for some odd ordinal $\gamma+1, D=D_{\gamma+1}$, and argue as we did earlier when building the generic object $G_{1}$ to construct in $V$ an $M$-generic object $H_{2}$ over $\mathbb{T}^{*}$.

Note now that since $N$ is given by an ultrapower of $M$ via a normal ultrafilter $\mathcal{U} \in M$ over $\delta$, Fact 2 of Section 1.2.2 of [6] tells us that $k_{2}^{\prime \prime} H_{2}$ generates an $N$-generic object $G_{2}^{*}$ over $k_{2}\left(\mathbb{T}^{*}\right)$. By elementarity, $k_{2}\left(\mathbb{T}^{*}\right)$ is the term forcing in $N$ defined with respect to $k_{2}\left(k_{1}\left(\mathbb{P}_{\delta}\right)_{\delta+1}\right)=\mathbb{P}_{\delta} * \dot{\mathbb{Q}}^{0}$. Therefore, since $j\left(\mathbb{P}_{\delta}\right)=k_{2}\left(k_{1}\left(\mathbb{P}_{\delta}\right)\right)=\mathbb{P}_{\delta} * \dot{\mathbb{Q}}^{0} * \dot{\mathbb{R}}^{0}, G_{2}^{*}$ is $N$-generic over $k_{2}\left(\mathbb{T}^{*}\right)$, and $G_{0} * G_{1}$ is $k_{2}\left(\mathbb{P}_{\delta} * \dot{\mathbb{S}}^{0}\right)$-generic over $N$, Fact 1 of Section 1.2 .5 of [6] (see also [8]) tells us that for $G_{2}=\left\{i_{G_{0} * G_{1}}(\tau): \tau \in G_{2}^{*}\right\}, G_{2}$ is $N\left[G_{0}\right]\left[G_{1}\right]$-generic over $\mathbb{R}^{0}$. Thus, in $V\left[G_{0}\right], j: V \rightarrow N$ lifts to $j: V\left[G_{0}\right] \rightarrow N\left[G_{0}\right]\left[G_{1}\right]\left[G_{2}\right]$, i.e., $V^{\mathbb{P}} \vDash$ " $\delta$ is $\lambda=\delta^{+\alpha}$ strongly compact". This completes the proof of Lemma 2.3.

Lemma 2.4. $V^{\mathbb{P}} \vDash$ "For any measurable cardinal $\delta$ and any $\alpha$ below the least beth fixed point above $\delta$, if $\delta^{+\alpha}$ is regular and $\delta$ is $\delta^{+\alpha}$ strongly compact but $\delta$ is not a limit of cardinals $\gamma$ which are $\delta^{+\alpha}$ strongly compact, then $\delta$ is $\delta+\alpha+1$ strong".

Proof. As in the proof of Lemma 2.3, we may assume without loss of generality that $\alpha>0$. This is since $\delta$ is measurable iff $\delta$ is $\delta^{+0}=\delta$ strongly compact iff $\delta$ is $\delta$ supercompact, so any elementary embedding $j: V \rightarrow M$ witnessing the measurability of $\delta$ which is generated by a normal ultrafilter over $\delta$ also witnesses that $\delta$ is $\delta+0+1=\delta+1$ strong.

We therefore suppose $\delta$ is as in the hypotheses of Lemma 2.4, i.e., in $V^{\mathbb{P}}$, $\alpha$ is below the least beth fixed point above $\delta, \delta^{+\alpha}$ is regular, $\delta$ is $\delta^{+\alpha}$ strongly compact, yet $\delta$ is not a limit of cardinals $\gamma$ which are $\delta^{+\alpha}$ strongly compact. Assume further towards a contradiction that in $V, \delta$ is a limit of cardinals $\gamma$ which are $\delta^{+\alpha}$ supercompact. Since any cardinal $\gamma$ which is $\delta^{+\alpha}$ supercompact is automatically $<\delta$ supercompact, by the remarks immediately following the proof of Lemma 2.1, we know that in $V^{\mathbb{P}}, \delta$ is a limit of cardinals $\gamma$ which are $<\delta$ supercompact. As $V^{\mathbb{P}} \vDash " \delta$ is $\delta^{+\alpha}$ strongly compact", by a theorem of DiPrisco [7], $V^{\mathbb{P}} \vDash$ "Any cardinal $\gamma$ which is either $<\delta$ supercompact or $<\delta$ strongly compact is $\delta^{+\alpha}$ strongly compact". Thus, in $V^{\mathbb{P}}$, $\delta$ is a limit of cardinals $\gamma$ which are $\delta^{+\alpha}$ strongly compact, a contradiction. 
We now know that in $V, \delta$ is not a limit of cardinals $\gamma$ which are $\delta^{+\alpha}$ supercompact. Also, as in the proof of Lemma 2.2, by writing $\mathbb{P}=\mathbb{P}_{\delta} * \dot{\mathbb{P}}^{\delta}$, it is readily seen that $\mathbb{P}$ "admits a gap at $\aleph_{1}$ and is mild with respect to $\delta$ ". By the results of [10] and [11], $\delta$ is therefore $\delta^{+\alpha}$ strongly compact in $V$. This means, by level by level equivalence between strong compactness and supercompactness, that in $V, \delta$ is $\delta^{+\alpha}$ supercompact. Hence, as in the proof of Lemma 2.3, $\Vdash_{\mathbb{P}_{\delta}}$ " $\dot{\mathbb{P}}^{\delta}$ is $\eta$-strategically closed for $\eta$ the least inaccessible above $\delta$ ". Therefore, since $\alpha$ is below the least beth fixed point above $\delta$, to show that $V^{\mathbb{P}} \vDash$ " $\delta$ is $\delta+\alpha+1$ strong", it suffices to show that $V^{\mathbb{P}_{\delta}} \vDash$ " $\delta$ is $\delta+\alpha+1$ strong".

To do this, we argue now in an analogous fashion to the proof of Lemma 2.2 of [1]. (See also the proof of Lemma 2.4 of [4].) We use for the proof of this lemma notation and terminology from the introductory section of [6]. Let $\lambda=\alpha+1$. Let $j: V \rightarrow M$ be an elementary embedding witnessing the $\delta+\lambda$ strongness of $\delta$ generated by a $\left(\delta, \delta^{+\lambda}\right)$-extender of width $\delta$ with $j(\delta)$ minimal so that $M \vDash " \delta$ is not $\delta+\lambda$ strong", and let $i: V \rightarrow N$ be the elementary embedding witnessing the measurability of $\delta$ generated by the normal ultrafilter $\mathcal{U}=\{x \subseteq \delta: \delta \in j(x)\}$. We then have the commutative diagram

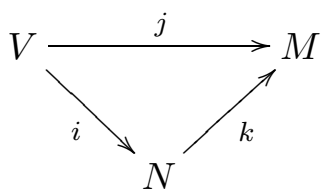

where $j=k \circ i$ and the critical point of $k$ is above $\delta$.

Observe that $M \vDash$ "No cardinal $\varrho \in\left(\delta, \delta^{+\lambda}\right]$ is measurable". This is since $V_{\delta+\lambda} \subseteq M$ and $V \vDash$ "Both $\alpha$ and $\lambda=\alpha+1$ are below the least beth fixed point above $\delta^{\prime \prime}$. Also, since $V_{\delta+\lambda} \subseteq M,\left(\delta^{+\lambda}\right)^{V}=\left(\delta^{+\lambda}\right)^{M}$. This means in $M$, the least measurable cardinal $\delta_{0}>\delta$ in the field of $j\left(\mathbb{P}_{\delta}\right)$ is so that $\delta_{0}>\delta^{+\lambda}$. In addition, it is the case that $\delta \notin \operatorname{field}\left(j\left(\mathbb{P}_{\delta}\right)\right)$. This is since, by choice of $\lambda$, $M \vDash$ "For every $\varrho<\alpha$ so that $\delta^{+} \varrho$ is regular, $\delta$ is $\delta^{+\varrho}$ supercompact and $\delta+\varrho+1$ strong". As $M \vDash " \delta$ is not $\delta+\lambda$ strong", there are no other degrees of either supercompactness or strongness that could affect whether $\delta$ is an element of field $\left(j\left(\mathbb{P}_{\delta}\right)\right)$.

Define now $f: \delta \rightarrow \delta$ by

$$
f(\varrho)=\text { The least measurable cardinal above } \varrho \text {. }
$$

We then have $\delta<\delta^{+\lambda}<j(f)(\delta)<\delta_{0}$. This last inequality is since the least measurable cardinal $\gamma$ above any $\varrho$ is not $\gamma+2$ strong, and by GCH in both $V$ and $M, \gamma$ is not $2^{\gamma}=\gamma^{+}$supercompact either. Thus, $\gamma$ is both $\gamma^{+0}=\gamma$ supercompact and $\gamma+0+1=\gamma+1$ strong and shows no further degrees of either supercompactness or strongness. 
Note that $M=\left\{j(g)(a): a \in\left[\delta^{+\lambda}\right]^{<\omega}, \operatorname{dom}(g)=[\delta]^{|a|}, g:[\delta]^{|a|} \rightarrow V\right\}$ $=\left\{k(i(g))(a): a \in\left[\delta^{+\lambda}\right]^{<\omega}, \operatorname{dom}(g)=[\delta]^{|a|}, g:[\delta]^{|a|} \rightarrow V\right\}$. By defining $\gamma=i(f)(\delta)$, we have $k(\gamma)=k(i(f)(\delta))=j(f)(\delta)>\delta^{+\lambda}$. This means $j(g)(a)=k(i(g))(a)=k\left(i(g)\left\lceil[\gamma]^{|a|}\right)(a)\right.$, i.e., $M=\left\{k(h)(a): a \in\left[\delta^{+\lambda}\right]^{<\omega}\right.$, $\left.h \in N, \operatorname{dom}(h)=[\gamma]^{|a|}, h:[\gamma]^{|a|} \rightarrow N\right\}$. By elementarity, we must have $N \vDash " \delta \notin \operatorname{field}\left(i\left(\mathbb{P}_{\delta}\right)\right)$ and $\delta<\gamma=i(f)(\delta)<\zeta=$ The least element of the field of $i\left(\mathbb{P}_{\delta}\right)-\delta$ ", since $M \vDash " k(\delta)=\delta$ is not in the field of $j\left(\mathbb{P}_{\delta}\right)$ and $k(\delta)=\delta<k(\gamma)=k(i(f)(\delta))=j(f)(\delta)<k(\zeta)=\delta_{0}$ ". Therefore, $k$ is generated by an $N$-extender of width $\gamma \in(\delta, \zeta)$.

Write $i\left(\mathbb{P}_{\delta}\right)=\mathbb{P}_{\delta} * \dot{\mathbb{Q}}^{0}$, where $\dot{\mathbb{Q}}^{0}$ is a term for the portion of $i\left(\mathbb{P}_{\delta}\right)$ whose field is composed of ordinals in the interval $[\delta, i(\delta))$. By our previous work, the field of $\dot{\mathbb{Q}}^{0}$ is actually composed of ordinals in the interval $(\delta, i(\delta))$, or more precisely, of ordinals in the interval $[\zeta, i(\delta))$. This means that if $G_{0}$ is once again $V$-generic over $\mathbb{P}_{\delta}$, the argument from Lemma 2.3 for the construction of the generic object $G_{1}$ can be applied here as well to construct in $V\left[G_{0}\right]$ an $N\left[G_{0}\right]$-generic object $G_{1}^{*}$ over $\mathbb{Q}^{0}$. Since $i^{\prime \prime} G_{0} \subseteq G_{0} * G_{1}^{*}, i$ lifts in $V\left[G_{0}\right]$ to $i: V\left[G_{0}\right] \rightarrow N\left[G_{0}\right]\left[G_{1}^{*}\right]$, and since $k^{\prime \prime} G_{0}=G_{0}$ and $k(\delta)=\delta$, $k$ lifts in $V\left[G_{0}\right]$ to $k: N\left[G_{0}\right] \rightarrow M\left[G_{0}\right]$. By Fact 3 of Section 1.2.2 of [6], $k: N\left[G_{0}\right] \rightarrow M\left[G_{0}\right]$ is also generated by an extender of width $\gamma \in(\delta, \zeta)$.

In analogy to the preceding paragraph, write $j\left(\mathbb{P}_{\delta}\right)=\mathbb{P}_{\delta} * \dot{\mathbb{Q}}^{1}$. By the last sentence of the preceding paragraph and the fact $\zeta$ is the least ordinal in the field of $\dot{\mathbb{Q}}^{0}$, we can use Fact 2 of Section 1.2.2 of [6] to infer that $H=\left\{p \in \mathbb{Q}^{1}: \exists q \in k^{\prime \prime} G_{1}^{*}[q \geq p]\right\}$ is $M\left[G_{0}\right]$-generic over $k\left(\mathbb{Q}^{1}\right)$. Thus, $k$ lifts in $V\left[G_{0}\right]$ to $k: N\left[G_{0}\right]\left[G_{1}^{*}\right] \rightarrow M\left[G_{0}\right][H]$, and we get the new commutative diagram

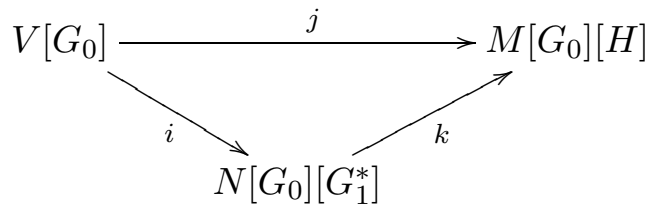

As in the proofs of Lemma 2.2 of [1] and Lemma 2.4 of [4], since $V_{\delta+\lambda} \subseteq$ $M$ and $G_{0} \in M\left[G_{0}\right][H]$, we can deduce that $\left(V\left[G_{0}\right]\right)_{\delta+\lambda} \subseteq M\left[G_{0}\right][H]$, i.e., that $j$ remains a $\delta+\lambda$ strong embedding after forcing with $\mathbb{P}_{\delta}$. This completes the proof of Lemma 2.4 .

Lemmas 2.1-2.4, along with the intervening remarks, complete the proof of Theorem 3.

3. Concluding remarks. In conclusion to this paper, we make several remarks. The first is that, as noted immediately following the statement of Theorem 3, larger degrees of level by level equivalence between strong compactness and strongness are possible, assuming the definition of our partial 
ordering $\mathbb{P}$ has been suitably modified. To see this, suppose the set or class $\mathfrak{D}$ used in the definition of $\mathbb{P}$ is changed to be the collection of $V$-measurable cardinals $\delta$ such that for some $\alpha$ below the second $V$-measurable cardinal above $\delta$, in $V, \delta^{+\alpha}$ is regular, $\delta$ is $\delta+\alpha+1$ strong, yet $\delta$ is not $\delta^{+\alpha}$ supercompact. $\mathbb{P}$ is then defined as before, using this new $\mathfrak{D}$ as the collection of measurable cardinals to which non-reflecting stationary sets of ordinals are added. Because our ground model $V$ satisfies level by level equivalence between strong compactness and supercompactness, no non-reflecting stationary sets of ordinals will be added to either the first or second measurable cardinal above any measurable cardinal. This is since these cardinals will not manifest any non-trivial degree of either strong compactness or supercompactness. Thus, as readers may easily verify for themselves, the proofs of Lemmas 2.1-2.4 suitably modified all remain valid, and produce a model $V^{\mathbb{P}}$ in which for any measurable cardinal $\delta$ and any $\alpha$ below the second measurable cardinal above $\delta$, if $\delta^{+\alpha}$ is regular, $\delta$ is $\delta^{+\alpha}$ strongly compact iff $\delta$ is $\delta+\alpha+1$ strong, except possibly if $\delta$ is a limit of cardinals $\gamma$ which are $\delta^{+\alpha}$ strongly compact.

There are limits, though, to the modifications that can be made to the set or class $\mathfrak{D}$ that still allow our methods of proof to go through. As an example, we may come to a point where the different non-reflecting stationary set forcings begin to interfere with one another, such as if a non-reflecting stationary set of ordinals has to be added to some cardinal $\gamma$, where $\delta<$ $\gamma<\lambda$ and $\delta$ is $\lambda$ supercompact. This would require a significant change in proof in the appropriate analogues of Lemmas 2.3 and 2.4 .

Thus, we conclude this paper by restating the questions we asked in Section 1, i.e., how much level by level equivalence between strong compactness and strongness is possible in a model containing supercompact cardinals, or more generally, in a model containing more than one strongly compact cardinal? In particular, is it possible to have a model containing more than one strongly compact cardinal in which there is unrestricted level by level equivalence between strong compactness and strongness, i.e., in which for every measurable cardinal $\delta$, if $\delta^{+\alpha}$ is regular, $\delta$ is $\delta^{+\alpha}$ strongly compact iff $\delta$ is $\delta+\alpha+1$ strong, except possibly if $\delta$ is a measurable limit of cardinals $\gamma$ which are $\delta^{+\alpha}$ strongly compact? (Recall that this question is answered in the case of a model containing one strongly compact cardinal by Theorem 1.) These seem like very difficult questions to answer, and constructing models in which these properties are true would require significantly different proof methods from those of this paper. In particular, in such models, not only would there not be level by level equivalence between strong compactness and supercompactness, but because every strong cardinal would have to be strongly compact and every supercompact cardinal has to have a normal measure concentrating on strong cardinals (see Lemma 2.1 of [4] for 
a proof of this fact), the Kimchi-Magidor property would fail as well, i.e., there would be non-supercompact strongly compact cardinals which are not measurable limits of strongly compact cardinals.

\section{References}

[1] A. Apter, On the level by level equivalence between strong compactness and strongness, J. Math. Soc. Japan 55 (2003), 47-58.

[2] - Strong compactness, measurability, and the class of supercompact cardinals, Fund. Math. 167 (2001), 65-78.

[3] - Supercompactness and measurable limits of strong cardinals, J. Symbolic Logic 66 (2001), 629-639.

[4] A. Apter and J. Cummings, Identity crises and strong compactness II: Strong cardinals, Arch. Math. Logic 40 (2001), 25-38.

[5] A. Apter and S. Shelah, On the strong equality between supercompactness and strong compactness, Trans. Amer. Math. Soc. 349 (1997), 103-128.

[6] J. Cummings, A model in which GCH holds at successors but fails at limits, ibid. 329 (1992), 1-39.

[7] C. A. Di Prisco and J. Henle, On the compactness of $\aleph_{1}$ and $\aleph_{2}$, J. Symbolic Logic 43 (1978), 394-401.

[8] M. Foreman, More saturated ideals, in: Cabal Seminar 79-81, Lecture Notes in Math. 1019, Springer, Berlin, 1983, 1-27.

[9] J. D. Hamkins, Destruction or preservation as you like it, Ann. Pure Appl. Logic 91 (1998), 191-229.

[10] —, Gap forcing, Israel J. Math. 125 (2001), 237-252.

[11] —, Gap forcing: Generalizing the Lévy-Solovay theorem, Bull. Symbolic Logic 5 (1999), 264-272.

[12] A. Kanamori, The Higher Infinite, Springer, Berlin, 1994.

[13] Y. Kimchi and M. Magidor, The independence between the concepts of compactness and supercompactness, circulated manuscript.

[14] T. Menas, On strong compactness and supercompactness, Ann. Math. Logic 7 (1974), 327-359.

Department of Mathematics

Baruch College of CUNY

New York, NY 10010 U.S.A.

E-mail: awabb@cunyvm.cuny.edu

http://faculty.baruch.cuny.edu/apter

Received 27 February 2003;

in revised form 13 July 2004 